\title{
DESCRIPTION D'UN NOUVEAU TYPE DE CAROTTIER POUR LES SÉDIMENTS TRES FLUIDES
}

\author{
par N. Giani ${ }^{1}$.
}

\begin{abstract}
Ce nouveau type de carottier est particulièrement conçu pour les sédiments fluides. Il est muni d'un tube de $8 \mathrm{~cm}$ de diamètre, se fermant aux deux extrémités. Ses performances sont supérieures à celles d'une benne Ekman dans les mêmes conditions. Les causes de cette différence sont analysées. Ce carottier permet en outre d'étudier la répartition verticale de la faune dans les sédiments. Les résultats préliminaires obtenus dans un lac de haute montagne sont présentés.
\end{abstract}

\section{Description of a new type of corer for very fluid sediments.}

This new corer was designed particularly for fluid sediments. It consists of a tube which is $8 \mathrm{~cm}$ in diameter and which closes a both ends. Its performance is superior to that of an Ekman grab in the same conditions. The reasons for this difference are analysed. It is also possible to study the vertical distribution of the fauna with this corer. Some preliminary results from a high moutain lake are given.

De nombreux appareils sont utilisés pour étudier les sédiments et la faune qui leur est associée. Ils peuvent se ramener à deux types: les uns râclent le substrat, les autres, tels les bennes munies de mâchoires et les tubes carottiers, le découpent. Plusieurs auteurs ont comparé les diverses méthodes d'étude et leurs mérites respectifs : Brinkhurst (1967), Brinkhurst et al. (1969), Chodorowski (1971), Flannagan (1970) et Kajak (1963 et 1971). Un bon échantillonneur doil pénétrer suffisamment dans le substrat et prélever une surface identique à toutes les profondeurs, être conçu de façon à minimiser l'onde de pression inévitable à son arrivée sur le fond, posséder un système de fermeture hermétique pour éviter toute perte lors de la remontée (Brinkhurst 1967, Kajak 1971 ). Rares sont les appareils qui répondent intégralement à ces exigences. Il n'existe pas en fait de méthode universelle de prélèvement: le choix de la technique d'échantillonnage dépend de la nature des sédiments el du groupe zoologique considéré (Flannagan, 1970$)$.

1. Laboratoire d'Hydrobiologie, Université Paul-Sabatier, 118, route de Narbonne, 31077 Toulouse Cedex. 


\section{1. - Le carottage des sédiments fluides.}

Pour des substrats mous, les carottiers semblent donner des résultats supérieurs à ceux des autres appareils; ils ne permettent cependant pas toujours un échantillonnage efficace du benthos des sédiments très fluides.

Si le sédiment pénètre dans le carottier grâce à l'action d'un piston (Brown 1956, Byrne et Kulm 1962, Livingstone 1955, Mackereth 1958, 1969, Rowley et Dalh 1956, Vallentyne 1955, Walker 1967 ), un tube de faible diamètre - 2 à $5 \mathrm{~cm}$ - doit être employé. Or, Mac Intyre (1970) a montré que les carottiers de faible section ne prélevaient pas la pellicule superficielle du sédiment, c'est-à-dire la strate la plus densément peuplée. De plus, la faible surface de prélèvement ne convient pas à l'échantillonnage du macrobenthcs. Ce type d'apparcil ne permet done que des études sédimentologiques.

Si le sédiment pénètre dans le tube sous la seulc action du poids du carottier, un plus grand diamètre (de $5 \mathrm{~cm}$ à $8 \mathrm{~cm}$ ) peut êtra employé (Brinkhurst et al. 1969, Kajak et al. 1965, Hakala 1971); Burke. (1969) utilise un diamètre atteignant $21 \mathrm{~cm}$. En présence d'un sédiment très fluide, tel que celui du lac de Port-Bielh à 2285 m dans les Pyrénées centrales, un système d'obturation hermétique de l'appareil devient indispensable. Seuls les carottiers de Jenkin-Mortimer (1941-1942), de Drzcimski (1967) et d'Elgmork (1965) sont pourvus d'un tel système. Mais le modèle de Mor!imer est lourd et d'un maniement trop difficile pour être utilisé sur des embarcations légères en montagne. Ceux de Drzcimski et d'Elgmork, plus faciles à manipuler, sont d'une étanchéité imparfaite.

Pour étudier les Oligochètes vivant dans les sédiments particulièrement fluides du lac de Port-Bielh, un nouveau type de carottier a dû être réalisé $e^{1}$ Il est muni d'un système de fermeture aux deux extrémités.

\section{2. - Description et manipulation.}

2.1. - Description. - L'appareil est représenté sur les figures 1, 2 et 3 . Il se compose essentiellement d'un tube (1) en altuglass de $8 \mathrm{~cm}$ de diamètre interne et de $60 \mathrm{~cm}$ de longueur qui est fixé à l'aide de deux boulons à ailettes (2). Le corps du carottier comprend ensuite trois systèmes mobiles, reliés à deux systèmes de déclenchement et servant à la double fermeture de ce tube.

Dans la partie supérieure (fig. 3 a) le ressort (3) pousse un couvercle (4) de même diamètre que le tube. Ceci permet le libre

1. Il m'est agréable de remercier ici mon frère, Serge Giani, qui a largement contribué à la conception et à la réalisation de cet appareil. 


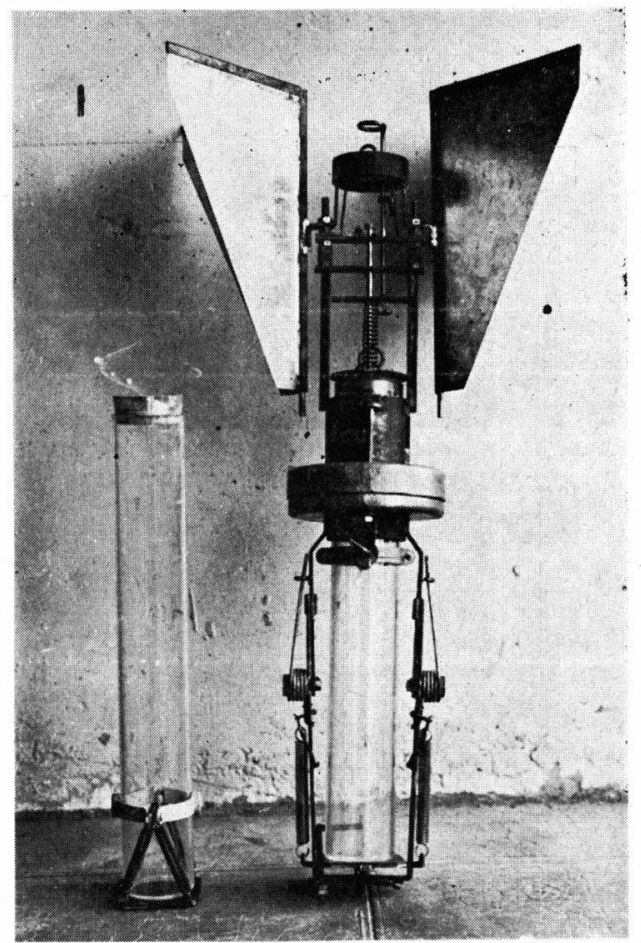

Fig. 1. - Vue générale de l'échantillonneur muni de son lest et des ailerons de stabilisation.

écoulement de l'eau lors de la descente et réduit la vague de pression (Brinkhurst 1969, Kajak 1963). La mise en place de l'étrier (5) contre les butées (6) par l'intermédiaire de deux puissants ressorts de rappel (7) amène en position le système d'obturation du bas du tube (fig. 2 ). Le rappel de l'étrier et la fermeture de l'extrémité supérieure du tube sont réalisés à partir du même dispositif de déclenchement (fig. 3 a). Lors du retour de l'étrier en position verticale, le choc du tube sur la tige (8) libère les deux ressorts (9) qui tirent la plaque caoutchoutée (10) vers le haut et réalisent ainsi la fermeture hermétique du tube.

2.2. - Manipulation. - Les ressorts (9) étant détendus on engage la boucle terminale du câble (11) dans le système de verrouillage (12) ce qui amène et maintient l'étrier en position horizontale (fig. 2). Par traction sur la tige (13) on enclenche le verrou (14) dans le cran (15); il est maintenu dans cette position par le ressort (16). On tend alors les deux ressorts (9) à l'aide des leviers (17) et le dispositif de fermeture inférieur est prêt à fonctionner. On arme ensuite le système supérieur en engageant la tête de 


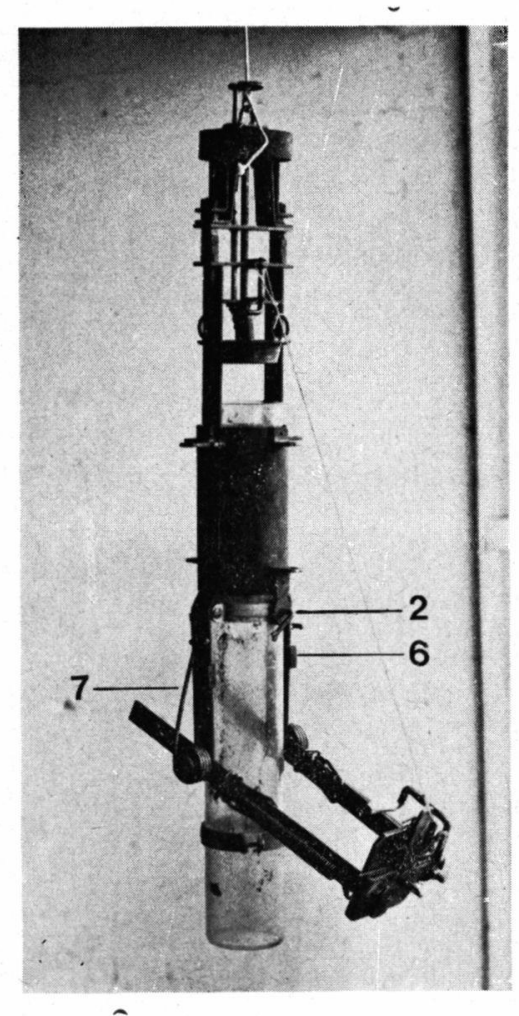

FIg. 2. - Vue de l'appareil en position de fonctionnement. 2. boulons à ailettes; 6. butées de l'étrier; 7. ressort de rappel de l'étrier.

l'axe (18) sur la butée (19); la tige (20), sciée en son milieu, donne la possibilité d'armer séparément l'étrier et le couvercle supérieur, le déclenchement restant simultané. L'appareil est ensuite descendu au bout d'une corde ou d'un câble ; le déclenchement se commande de la surface par l'intermédiaire d'un messager. Compte tenu de la fluidité des sédiments considérés il n'est pas nécessaire de descendre l'appareil très rapidement car il s'enfonce très facilement sous l'action de son propre poids (6 kg dans l'air). Un lest (15 kg dans l'air) formé de quatre demi-couronnes de plomb fixées sur le porte tube (23) à l'aide de deux boulons (21) peut également être utilisé. Lorsque, pour des sédiments plus durs, une descente rapide est nécessaire, on peut adapter des volets de stabilisation qui évitent à l'appareil de se coucher.

Après la remontée, le sédiment est versé dans un récipient adéquat. Pour conserver le prélèvement intact il conviendra de dévisser les boulons à ailettes (2) et de désolidariser la plaque 
(10) de l'étrier à l'aide du verrou (22) afin d'ôter le tube. Auparavant la plaque (10) est fixée au tube par l'intermédiaire de quatre tendeurs (fig. 1) qui maintiennent le tube hermétiquement clos.
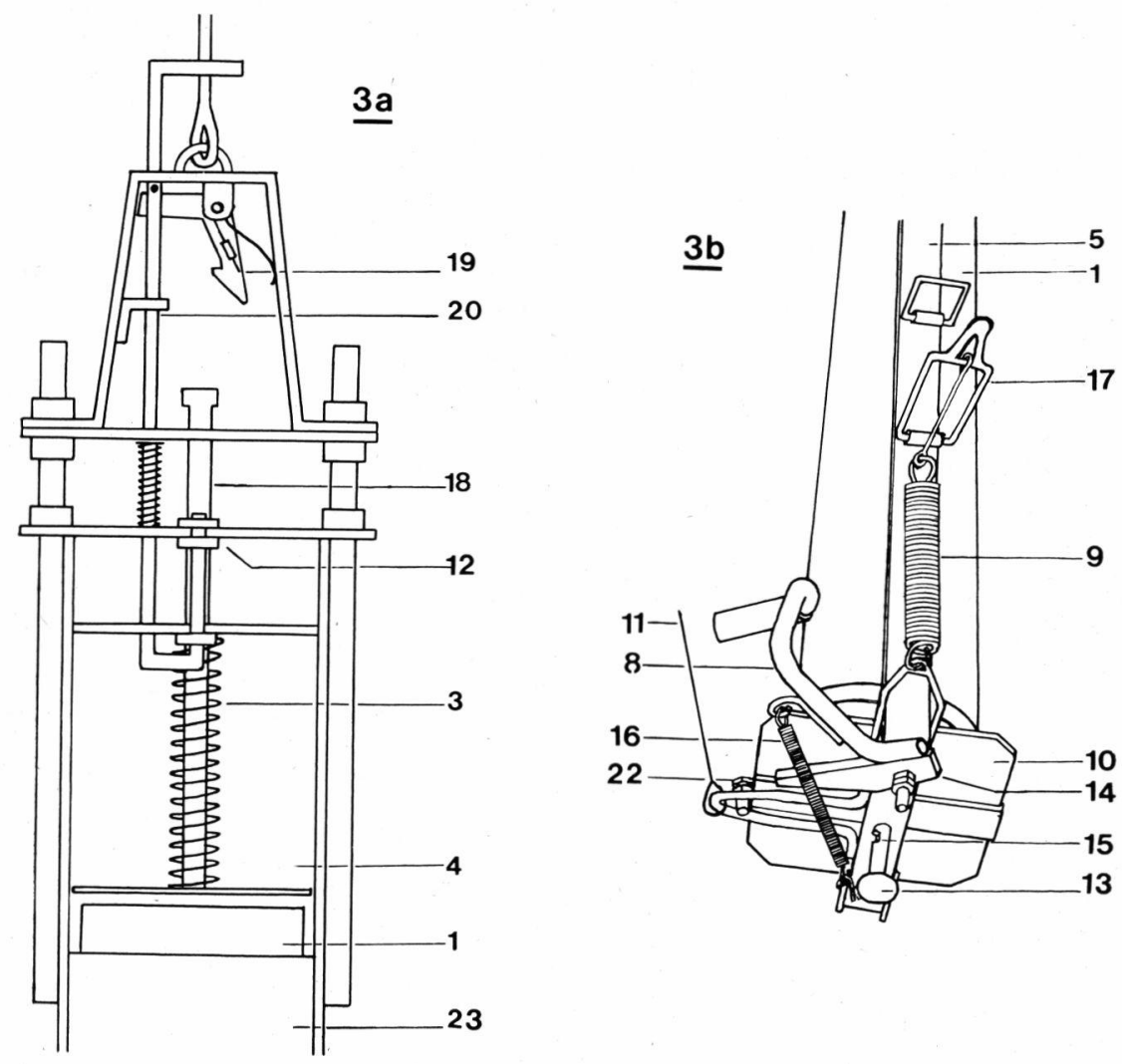

FIG. 3. - Détails de l'appareil. 3 a. partie supérieure; $3 \mathrm{~b}$. partie inférieure; 3. ressort de fermeture du couvercle; 4. couvercle; 8 . tige de déclenchement; 9. ressorts de fermeture; 10 . plaque caoutchoutée; 11. câble de l'étrier; 12. verrou; 13. tige crantée; 14. verrou inférieur; 15. cran de sécurité; 16. ressort de rappel; 17. levier; 18 . axe du couvercle; 19. butée; 20. tige sciée; 21. boulons de fixation du lest; 22 . verrou de la plaque; 23 . porte-tube.

\section{3. - Résultats préliminaires.}

Ce nouvel appareil nous a permis d'étudier la répartition verticale de la faune dans le substrat ainsi que les caractéristiques physico-chimiques du sédiment. Une analyse détaillée de ces résultats sera publiée ultérieurement (Giani et Lucas en préparation). Les premiers résultats montrent que 76,9 \% du benthos vivent dans les cinq premiers centimètres et près de $90 \%$ dans les dix 
premiers centimètres. Cependant la pénétration en profondeur varie selon les organismes considérés. Ainsi, $25 \%$ des Oligochètes viven ${ }^{\dagger}$ encore au-delà de $10 \mathrm{~cm}$, et jusqu'à des profondeurs pouvant atteindre $45-50 \mathrm{~cm}$. Chironomus plumosus ne s'enfonce pas aussi profondément que les Oligochètes mais $18 \%$ de sa population colonisent la zone comprise entre 10 et $20 \mathrm{~cm}$. Parmi les Oligochètes, Tubifex tubifex vit plus profondément que Peloscolex pyrenaicus. La quasi totalité des Crustacés et des 'Tany'arsini est concentrée dans le niveau supéricur des sédiments et notamment dans les cinq premiers centimètres. Le tube carottier permet donc d'obtenir, outres des estimations de densité, une localisation précise de la faune dans le sédiment, ce qui n'est pas possible avec la benne Ekman.

\section{4. - Comparaison entre le carottier et la benne Ekman.}

Afin de tester la validité de notre tube carottier nous l'avons comparé avec la benne Ekman qui est l'apparcil de prélèvement le plus largement utilisé pour l'étude du benthos.

Le premier essai parallèle des deux appareils a été réalisé à $16 \mathrm{~m}$ de profondeur dans le lac de Port-Bielh; il porte sur 5 bennes et 8 carottages. Le deuxième essai, effectué à $19 \mathrm{~m}$, se compose d'une série de 6 bennes et 9 carottages.

4.1. - RÉsultats. - Les résultats moyens concernant les principaux groupes systématiques sont reportés dans le tableau suivant (pour faciliter la comparaison nous avons ramené le prélèvement par carottier à la superficie de la benne).

Tabledu I. - Comparaison des résultats obtenus à l'aide de la benne Ekman et du tube carottier (moyenne et limites de confiance au seuil $P=0,05$ ).

\begin{tabular}{lcccc}
\hline & & \multicolumn{2}{c}{$19 \mathrm{~m}$} \\
\hline & Ekman & Carottier & Ekman & Carottier \\
\hline & & & & \\
Crustacés & $21,2 \pm 33,3$ & $289,9 \pm \mathbf{5 9 , 5}$ & $21,5 \pm 16,1$ & $471,13 \pm 145,7$ \\
Oligochètes & $\mathbf{3 5 , 2} \pm \mathbf{6 , 2 2}$ & $120,7 \pm \mathbf{4 0 , 2}$ & $38,6 \pm 13,1$ & $\mathbf{2 1 9 , 5} \pm \mathbf{3 3 , 9}$ \\
Chironomes & $26,8 \pm 37,3$ & $\mathbf{1 3 4 , 6 \pm 3 2 , 7}$ & $13,2 \pm 9,7$ & $170,7 \pm \mathbf{5 9 , 1}$ \\
& & & & \\
\hline
\end{tabular}

Les résultats obtenus à l'aide du carottier sont supérieurs à ceux fournis par la benne.

Afin de préciser ces différences, nous avons comparé, espèce par espèce, les données fournies par les deux appareils, à l'aide du test $\mathbf{U}$ de Mann-Whitney (tableau II). Pour les formes peu abondantes à ces profondeurs, telles Sialis lutaria, Cricotopus pirifer les résultats ne diffèrent pas significativement. Pour Psectrocladius sordidellus, Zavrelimyia melanura, Candona neglecta, la différence 
est significative à $16 \mathrm{~m}$ où ces espèces sont abondantes; elle ne l'est pas à $19 \mathrm{~m}$ où ces trois espèces sont rares. C'est l'inverse pour les Tanytarsini, dont la densité à $16 \mathrm{~m}$ est inférieure à celle à $19 \mathrm{~m}$. Pour les espèces abondantes les différences sont toujours significatives.

Tableau II. - Comparaison des densités moyennes yar m² obtenues à l'aide de la benne Ekman et du carottier.

* Test U de Mann-Whitney significatif au seuil de $5 \%$ ( $\mathrm{U} \leqslant 6$ pour $16 \mathrm{~m}$; $\mathrm{U} \leqslant 10$ pour $19 \mathrm{~m}$ ).

\begin{tabular}{|c|c|c|c|c|c|c|}
\hline & & $16 \mathrm{~m}$ & & & $19 \mathrm{~m}$ & \\
\hline & $\begin{array}{r}\text { Benne } \\
\text { Ekman }\end{array}$ & Carottier & $\mathrm{U}$ & $\begin{array}{l}\text { Benne } \\
\text { Ekman }\end{array}$ & Carottier & $\mathrm{U}$ \\
\hline \multicolumn{7}{|l|}{ Chironomides } \\
\hline P. sordidellus & 578,5 & 2886,5 & $6^{*}$ & 14,7 & 149,25 & 18,5 \\
\hline C. pirifer & 89 & 398 & $2^{\star}$ & - & - & - \\
\hline Z. melanura & 71,2 & 471,6 & $11,5^{\star}$ & 29,4 & 328,3 & 10,5 \\
\hline C. plumosus & 267 & 1910,4 & $6^{*}$ & 154,2 & 1596,9 & $5^{*}$ \\
\hline Tanytarsini & 186,9 & 322,4 & 14 & 418,8 & 5467,5 & $0^{*}$ \\
\hline \multicolumn{7}{|l|}{ Oligochètes } \\
\hline T. tubifex & 1343,9 & 5193,9 & $0^{*}$ & 1519,7 & 5248,6 & $0^{\star}$ \\
\hline$P$. pyrenaicus & $\mathbf{2 0 4 , 7}$ & 23,8 & 13,5 & 199,4 & 4552,1 & $0^{*}$ \\
\hline L. variegatus & 17,8 & 99.5 & 14 & - & - & - \\
\hline \multicolumn{7}{|l|}{ Ostracodes } \\
\hline C. lacustris & 293,7 & 1168 & $4^{*}$ & 852,2 & 14681,1 & $0^{\star}$ \\
\hline C. neglecta & 542,9 & 4139,2 & $2^{\star}$ & 29,4 & 318,4 & 17,5 \\
\hline \multicolumn{7}{|l|}{ Copépodes } \\
\hline C. staphylinus & 17,8 & 7164 & $0^{*}$ & 80,8 & 3452,2 & $0^{*}$ \\
\hline \multicolumn{7}{|l|}{ Cladocères } \\
\hline E. lamellatus & 71,2 & 322,4 & 7,5 & - & - & - \\
\hline Divers & 17,8 & - & - & 29,4 & 1243,7 & $4^{*}$ \\
\hline \multicolumn{7}{|l|}{ Mégaloptères } \\
\hline S. lutaria & 17,8 & 123,4 & 14 & 29,4 & 149,2 & 24,5 \\
\hline Nématodes & 53,4 & 322,4 & $6^{\star}$ & 14,7 & 183,8 & 24,5 \\
\hline Planaires & - & - & - & 14,7 & 558,2 & $4^{*}$ \\
\hline
\end{tabular}

A $19 \mathrm{~m}$, les différences entre les deux techniques d'échantillonnage portent essentiellement sur les organismes vivant à la surface du sédiment: Planaires, Crustacés, Peloscolex pyrenaicus, Tanytarsini. Ceci laisse présager que la benne s'enfonce trop profondément dans le substrat particulièrement mou et dépourvu de végétation à cette profondeur. Afin de vérifier ceci, le 25-11-1973 nous avons effectué, dans cette zone du lac une série de trois prélèvements à la benne (tableau III). La benne A a été volontairement descendue lentement et retenue lors de son arrivée sur le substrat afin de limiter sa pénétration. Ceci s'est traduit par la récolte d'un plus grand nombre de Peloscolex pyrenaicus, de Crustacés et de Tanytarsini que dans les bennes manipulées normalement. 
TABleau 3. - Résultats des 3 bennes prélevées le 25-11-1973. La benne A a été retenue lors de son arrivée sur le substrat.

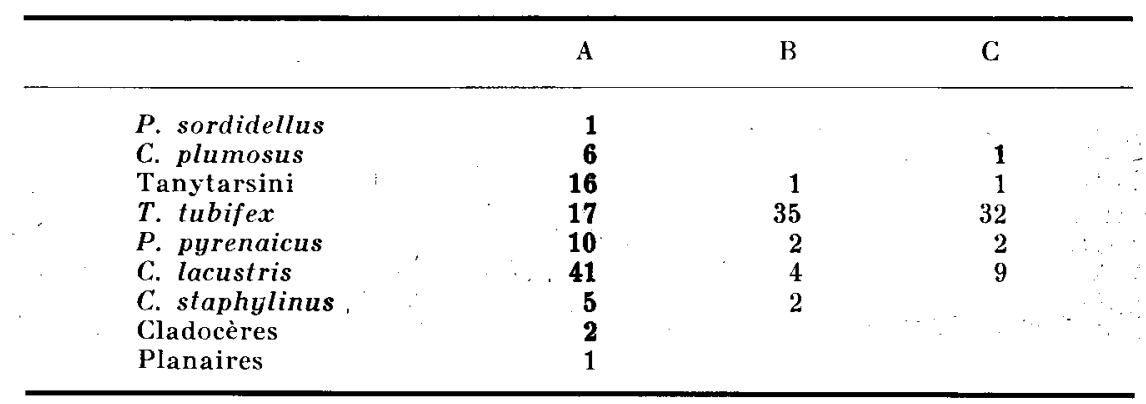

4.2. - Discussion. - Hamilton, Burton et Flannagan (1970) ont déjà noté la supériorité des carottiers dans les sédiments mous. Digerfeldt et Lettevall (1969), Kajak (1971) ont signalé la variabilité des résultats fournis par la benne Ekman qui, s'enfonçant plus ou moins profondément dans le substrat, peut perdre une partie de son contenu par le haut.

Dans le lac de Port-Bielh, les sédiments de la zone comprise entre 16 et $19 \mathrm{~m}$ - zone où les Oligochètes sont l'élément dominant du macrobenthos - sont particulièrement fluides; la benne qui mesure seulement $15 \mathrm{~cm}$ de hauteur s'enfonce trop dans le substrat. Entre 10 et $16 \mathrm{~m}$, la densité de la prairie de Nitella flexilis gêne la pénétration du carottier et ne nous a pas permis la comparaison. La zone littorale, formée d'éboulis granitiques, est difficile, impossible même, à prospecter par les moyens de prélèvements classiques.

Comme l'a remarqué Flannagan (1970), la benne vire de gauche à droite lors de la remontée. Les volets supérieurs tendent alors à s'ouvrir, entraînant une perte de sédiment lorsque la benne est trop pleine. Ceci peut être une cause d'erreur supplémentaire dans le cas considéré. Un verrouillage automatique de la benne doit donc se produire au niveau du substrat; en effet une autre solution telle que la mise en place d'un filet sur l'ouverture supérieure créerait une onde de pression.

Cette dernière est une des principales causes d'erreur dans les prélèvements benthiques (Lindroth 1935, Thorson 1957 in Wigley 1967, Lie et Pamatmat 1965, Kajak 1971). La sévérité des remous ainsi créés au niveau du substrat est en rapport direct avec la restriction de la circulation de l'eau à travers l'appareil d'une part et la vitesse de descente d'autre part. Notre tube carottier descendu à vitesse modérée minimise l'onde de pression et son double système de fermeture évite toute perte lors de la remontée. Toutefois comme le conseille Flannagan (1970) nous ne surestimerons pas les limites de notre appareil et « resterons très prudents en inter- 
prétant les résultats «quantitatifs» des travaux précédemment effectués ».

\section{TRAVAUX GITÉS}

Brinkmurst (R. O.). 1967. - Sampling the benthos. Great Lakes Inst., Prog. Rep. 32, Univ. Toronto: 7 p.

Brinkhurst (R. O.), Chui (K. E.) et Batoosingh (E.). 1969, - Modifications in sampling procedures as applied to studies on the Bacteria and Tubificid Oligochaetes inhabiting aquatic sediments. J. Fish. Res. Bd. Can., 26 : $2581-2593$.

Brown (S. R.). 1956. - A piston sampler for surface sediments of lake deposits. Ecology, $37: 611-613$.

Burke (J. C.). 1969. — A sediment coring device of $21 \mathrm{~cm}$ diameter with sphincter core retainer. Limnol. Oceanog., 13 : 714-718.

ByRne (J. V.) et Kulm (L. D.). 1962. - An inexpensive lightweight piston corer. Limnol. Oceanog., 7 : 106-108.

Chodorowsiki (A.). 1971. - L'échantillonnage des peuplements d'invertébrés des eaux continentales stagnantes. In LAMotTe (M.) et Bouritière (F.). Problèmes d'écologie: l'échantillonnage des peuplements animaux des milieux aquatiques. Paris, Masson et $\mathrm{C}^{\text {ie }}$, éd., 294 p.

Digerfeld (G.) et Lettevall. (U.). 1969. - A new type of sediment sampler. Geologiska Föreningens Stockholm Förhandlingar, 91 : 399-406.

ElgmoR K (K.). 1962. - A bottom sampler for soft mud. Hydrobiologia, $20: 167-172$.

FlanNaGan (J. F.). 1970. -- Efficiencies of various grabs and corers in sampling freshwater benthos. J. Fish Res. Bd. Can., 27 : 1691-1700.

HaKala (I.). 1971. - A new model of the Kajak bottom sampler, and other improvements in the zoobenthos sampling technique. Ann. Zool. Fenn., 8 : 422-426.

Hamilton (A. L.), Burton (W.) et Fuannagan (J. F.). 1970. - A multiple corer for sampling profundal benthos. J. Fish Res. Bd. Can., 27 : $1867-1869$.

KaJAK (Z.). 1963. - Analysis of quantitative benthic methorls. Ekol Polska, $11: 1-56$.

KaJAK (Z.). 1971. - Benthos of standing water. In Edmondson (W. T.) et Winberg (G. G.). A manual on methods for the assessment of secondary Productivity in Fresh Waters I.B.P. Handbook $n^{\circ} 17$.

Kajak (Z.), Kacprzak (K.) et Polkowski (R.). 1965. - Chwytacz rurowy do Pobierania prób Ina. Ekol. Pol. B, 11 : 159-165.

Lie (U.) et Pamatmat (M. M.). 1965. - Digging characteristics and sampling efficiency of the $0,1 \mathrm{~m}^{2}$ Van Veen grab. Limnol. Oceanog., $10: 379-384$.

Livingstone (D. A.). 1955. - A lightweight piston sampler for lake deposits. Ecology, 36 : 137-139.

Mac Intyre (A. D.). 1971. - Deficiency of gravity corers for sampling microbenthos and sediments. Nature, Lond., $231: 260$.

MACKeRETh (F. J.H.). 1958. - A portable core sampler for lake teposits. Limnol. Oceanog., $3: 181-191$.

MACKeneth (F.J.H.). 1969. - A short core sampler for subaqueous deposits. Limnol. Oceanog., 14 : 145-151. 
Mortimer (C. H.). 1941-42. - The exchange of dissolved substances between mud and water in lakes. J. Ecol., $29: 280-320 ; 30: 147-201$.

Rowley (J. R.) et DalH (A. O.). 1956. - Modifications in desing and use of the Livingstone piston sampler. Ecology, 37 : 849-851.

VAllentyne (J. R.). 1955. - A modification of the Livingstone piston sampler for lake deposits. Ecology, 36 : 139-141.

WALker (B.). 1967. - A diver operated pneumatic core sampler. Limnol. Oceanog., 12 : 144-147.

Wigley (R. L.). 1967. - Comparative efficiencies of Van Veen and Smith - Mc Intyre grab samplers as revealed by motion pictures. Ecology, $48: 168-169$. 